\title{
Regularity Criteria for a Coupled Navier-Stokes and Q-Tensor System
}

\author{
Jishan Fan ${ }^{1}$ and Tohru Ozawa ${ }^{2}$ \\ ${ }^{1}$ Department of Applied Mathematics, Nanjing Forestry University, Nanjing 210037, China \\ ${ }^{2}$ Department of Applied Physics, Waseda University, Tokyo 169-8555, Japan
}

Correspondence should be addressed to Tohru Ozawa; txozawa@waseda.jp

Received 14 September 2012; Accepted 7 April 2013

Academic Editor: Jens Lorenz

Copyright (C) 2013 J. Fan and T. Ozawa. This is an open access article distributed under the Creative Commons Attribution License, which permits unrestricted use, distribution, and reproduction in any medium, provided the original work is properly cited.

We study a system describing the evolution of a nematic liquid crystal flow. The system couples a forced Navier-Stokes system describing the flow with a parabolic-type system describing the evolution of the nematic crystal director fields ( $Q$-tensors). We prove some regularity criteria for the local strong solutions. However, we do not provide estimates on the rates of increase of high norms.

\section{Introduction}

We consider the following coupled Navier-Stokes and Qtensor system [1-4]:

$$
\begin{gathered}
\partial_{t} Q+u \cdot \nabla Q+Q \Omega-\Omega Q \\
=\Delta Q-a Q+b\left(Q^{2}-\frac{\mathbb{}}{d} \operatorname{tr}\left(Q^{2}\right)\right)-c Q \operatorname{tr}\left(Q^{2}\right), \\
\partial_{t} u+u \cdot \nabla u+\nabla \pi=\Delta u-\operatorname{div}(\nabla Q \odot \nabla Q) \\
+\operatorname{div}(Q \Delta Q-\Delta Q Q), \\
\operatorname{div} u=0, \\
(Q, u)(x, 0)=\left(Q_{0}, u_{0}\right) \quad \text { in } \mathbb{R}^{d}(d=2,3) .
\end{gathered}
$$

Here the unknowns $u, \pi$, and $Q$ denote the velocity field of the fluid, the pressure, and the order parameter, respectively. A $Q$-tensor is a symmetric and traceless $d \times d$-matrix, $\Omega$ := $(1 / 2)\left(\nabla u-(\nabla u)^{T}\right), a \in \mathbb{R}, b>0$ and $c>0$ are physical constants, $d$ is the space dimension, $(\nabla Q \odot \nabla Q)_{i j}:=$ $\operatorname{tr}\left(\partial_{i} Q \partial_{j} Q\right), \operatorname{tr} Q:=\sum_{i=1}^{d} Q_{i i}$, and thus $\operatorname{tr}\left(Q^{2}\right)=\sum_{i, j=1}^{d} Q_{i j}^{2}$.
When $Q \equiv 0,(2)$ and (3) are the well-known NavierStokes system, for which Kozono et al. [5] and Kozono and Shimada [6] proved the well-known regularity criteria

$$
\begin{gathered}
u \in L^{2 /(1-s)}\left(0, T ; \dot{B}_{\infty, \infty}^{-s}\right), \quad 0<s<1, \\
u \in L^{2}\left(0, T ; \dot{B}_{\infty, \infty}^{0}\right), \\
\omega:=\operatorname{curl} u \in L^{1}\left(0, T ; \dot{B}_{\infty, \infty}^{0}\right),
\end{gathered}
$$

where $\dot{B}_{p, q}^{s}$ denotes the homogeneous Besov spaces [7].

Very recently, Paicu and Zarnescu [8] proved the existence of global-in-time weak solutions in 3-dimensional space and of smooth solutions in 2-dimensional space. The aim of this paper is to study the regularity criteria.

If one formally takes $Q:=s_{+}(n \otimes n-(1 / 3) \square)$, with $s_{+}$a constant, then the equations reduce to the generally accepted equations of Leslie [9], which have been studied in [10-15]. We will prove the following.

Theorem 1. Let $u_{0} \in H^{1}, Q_{0} \in H^{2}$, div $u_{0}=0$ in $\mathbb{R}^{d}$ with $d=2$, 3. Let $(u, Q)$ be a unique strong solution in $L^{\infty}\left(0, T_{1} ; H^{1}\right) \cap L^{2}\left(0, T_{1} ; H^{2}\right) \times L^{\infty}\left(0, T_{1} ; H^{2}\right) \cap L^{2}\left(0, T_{1} ; H^{3}\right)$ with $0<T_{1}<T<\infty$. 
(i) If $d=2,3$ and $u$ satisfies one of the conditions (5), (6), or (7) and $\nabla Q$ satisfies

$\nabla Q \in L^{p}\left(0, T ; L^{q}\right) \quad$ with $\frac{2}{p}+\frac{d}{q}=1, d<q \leq \infty$,

for some finite $T<\infty$, then the solution $(u, Q)$ can be extended beyond $T>0$.

(ii) If $d=3$ and $u$ satisfies

$\nabla u \in L^{p}\left(0, T ; L^{q}\right) \quad$ with $\frac{2}{p}+\frac{3}{q}=2,2<q \leq 3$,

for some finite $T<\infty$, then the solution $(u, Q)$ can be extended beyond $T>0$.

Remark 2. By the well-known inequality $\|$ curl $u \|_{L^{q}} \leq$ $\|\nabla u\|_{L^{q}} \leq C\|\operatorname{curl} u\|_{L^{q}}$, the condition (9) can be replaced by

$$
\omega:=\operatorname{curl} u \in L^{p}\left(0, T ; L^{q}\right) \quad \text { with } \frac{2}{p}+\frac{3}{q}=2, \frac{3}{2}<q \leq 3 .
$$

It has been proved in [8] that the system (1)-(4) has a Lyapunov functional:

$$
\begin{aligned}
E(t):= & \int \frac{1}{2} u^{2}+\frac{1}{2}|\nabla Q|^{2}+\frac{a}{2} \operatorname{tr}\left(Q^{2}\right)-\frac{b}{3} \operatorname{tr}\left(Q^{3}\right) \\
& +\frac{c}{4} \operatorname{tr}^{2}\left(Q^{2}\right) d x
\end{aligned}
$$

which satisfies

$$
\begin{gathered}
\frac{d}{d t} E=-\int|\nabla u|^{2} d x \\
-\int \operatorname{tr}\left(\Delta Q-a Q-b\left(Q^{2}-\frac{\square}{d} \operatorname{tr}\left(Q^{2}\right)\right)\right. \\
\left.-c Q \operatorname{tr}\left(Q^{2}\right)\right)^{2} d x \leq 0,
\end{gathered}
$$

from which we easily obtain [8]

$$
\begin{gathered}
u \in L^{\infty}\left(0, T ; L^{2}\right) \cap L^{2}\left(0, T ; H^{1}\right), \\
Q \in L^{\infty}\left(0, T ; H^{1}\right) \cap L^{2}\left(0, T ; H^{2}\right) .
\end{gathered}
$$

When $d=2,(13)$ give [8]

$$
\begin{gathered}
u \in L^{4}\left(0, T ; L^{4}\right) \subset L^{2 /(1-1 / 2)}\left(0, T ; \dot{B}_{\infty, \infty}^{-1 / 2}\right) \\
\nabla Q \in L^{4}\left(0, T ; L^{4}\right)
\end{gathered}
$$

thus (5) and (8) hold true; this proves the existence of globalin-time strong solutions when $d=2$. In [8], this result was proven by complicated Littlewood-Paley theory, Bony's paraproduct decomposition, and the logarithmic Sobolev inequality. The purpose of this paper is to make the argument in [8] much simpler. However, in [8], they obtained in addition the rate of increase of high norms.

Our proof uses an energy method and relies on a simple $L^{\infty}$ estimate of $Q$ and the following cancellation property:
Lemma 3 (see $[8]$ ). Let $\widetilde{Q}, Q: \mathbb{R}^{d} \rightarrow \mathbb{R}^{d \times d}$ be symmetric matrix-valued functions and let $\Omega_{\alpha \beta}:=(1 / 2)\left(\partial_{\beta} u_{\alpha}-\partial_{\alpha} u_{\beta}\right) \epsilon$ $\mathbb{R}^{d \times d}$ be smooth and decaying and sufficiently fast at infinity (so that one can integrate by parts without boundary terms). Then

$$
\begin{aligned}
& \int \operatorname{tr}((\Omega \widetilde{Q}-\widetilde{Q} \Omega) \Delta Q) d x \\
& \quad-\int \sum_{\alpha, \beta, \gamma} \partial_{\beta}\left(\widetilde{Q}_{\alpha \gamma} \Delta Q_{\gamma \beta}-\Delta Q_{\alpha \gamma} \widetilde{Q}_{\gamma \beta}\right) u_{\alpha} d x=0 .
\end{aligned}
$$

\section{Proof of Theorem 1}

This section is devoted to the proof of Theorem 1 . Since it is easy to prove that there are $T_{0}>0$ and a unique strong solution $(u, Q)$ to the problem $(1)-(4)$ in $\left(0, T_{0}\right]$, we only need to prove a priori estimates.

First, we prove the following key estimate:

$$
\|Q\|_{L^{\infty}\left(0, T ; L^{\infty}\right)} \leq C
$$

To prove (16), we multiply (1) by $2 p Q \operatorname{tr}^{p-1}\left(Q^{2}\right)$ and take the trace to obtain

$$
\begin{aligned}
\left(\partial_{t}+\right. & u \cdot \nabla) \operatorname{tr}^{p}\left(Q^{2}\right) \\
= & 2 p \Delta Q_{\alpha \beta} Q_{\alpha \beta} \operatorname{tr}^{p-1}\left(Q^{2}\right)-2 p a \operatorname{tr}^{p}\left(Q^{2}\right) \\
& +2 p b \operatorname{tr}\left(Q^{3}\right) \operatorname{tr}^{p-1}\left(Q^{2}\right)-2 p c \operatorname{tr}^{p+1}\left(Q^{2}\right) .
\end{aligned}
$$

Let us observe that for $Q$, a traceless, symmetric, $3 \times 3$ matrix, we have

$$
\operatorname{tr}\left(Q^{3}\right) \leq \frac{3 \epsilon}{8} \operatorname{tr}^{2}\left(Q^{2}\right)+\frac{1}{\epsilon} \operatorname{tr}\left(Q^{2}\right), \quad \forall \epsilon>0 .
$$

Integrating over $\mathbb{R}^{d}$, integrating by parts, and using (3), (18), and the assumption $c>0$, we obtain

$$
\begin{aligned}
& \frac{d}{d t} \int \operatorname{tr}^{p}\left(Q^{2}\right) d x \leq-2 p \int \nabla Q_{\alpha \beta} \nabla Q_{\alpha \beta} \operatorname{tr}^{p-1}\left(Q^{2}\right) d x \\
& \quad-4 p(p-1) \int \partial_{\gamma} Q_{\alpha \beta} Q_{\alpha \beta} \partial_{\gamma} Q_{\delta \lambda} Q_{\delta \lambda} \operatorname{tr}^{p-2}\left(Q^{2}\right) d x \\
& \quad+c p \int \operatorname{tr}^{p}\left(Q^{2}\right) d x \\
& \leq c p \int \operatorname{tr}^{p}\left(Q^{2}\right) d x,
\end{aligned}
$$

which gives

$$
\left(\int \operatorname{tr}^{p}\left(Q^{2}\right) d x\right)^{1 /(2 p)} \leq e^{C T}\left(\int \operatorname{tr}^{p}\left(Q_{0}^{2}\right) d x\right)^{1 /(2 p)}
$$

with $C$ independent of $p$.

Thanks to a simple lemma in [16, Page 102], we take $p \rightarrow$ $\infty$ in (20), this proves (16). 
Taking $\Delta$ to (1), testing scalarly by $\Delta Q$, and using (3); we find that

$$
\begin{aligned}
& \frac{1}{2} \frac{d}{d t} \int|\Delta Q|^{2} d x+\int|\nabla \Delta Q|^{2} d x \\
& =\int(\Delta \Omega Q-Q \Delta \Omega): \Delta Q d x \\
& \quad+\int(\Omega \Delta Q-\Delta Q \Omega): \Delta Q d x \\
& \quad+2 \sum_{i} \int\left(\partial_{i} \Omega \partial_{i} Q-\partial_{i} Q \partial_{i} \Omega\right): \Delta Q d x \\
& \quad-\int \Delta u \cdot \nabla Q: \Delta Q d x \\
& \quad+2 \sum_{i, j} \int \partial_{i} u_{j} \partial_{j} \partial_{i} Q: \Delta Q d x \\
& \quad+\int \Delta\left[-a Q+b\left(Q^{2}-\frac{\mathbb{1}}{d} \operatorname{tr}\left(Q^{2}\right)\right)-c Q \operatorname{tr}\left(Q^{2}\right)\right]: \Delta Q d x \\
& =: I_{1}+I_{2}+I_{3}+I_{4}+I_{5}+I_{6} .
\end{aligned}
$$

Here "testing scalarly by $\Delta Q$ " means multiplying with respect to the Frobenius inner product of matrices, $A: B=\operatorname{tr}(A B)$ and integrating over $\mathbb{R}^{d}$.

Testing (2) by $-\Delta u$ and using (3), we infer that

$$
\begin{aligned}
\frac{1}{2} \frac{d}{d t} \int|\nabla u|^{2} d x+\int|\Delta u|^{2} d x \\
=\int(u \cdot \nabla) u \cdot \Delta u d x+\int \operatorname{div}(\nabla Q \odot \nabla Q) \Delta u d x \\
\quad-\int \operatorname{div}(Q \Delta Q-\Delta Q Q) \Delta u d x \\
=: J_{1}+J_{2}+J_{3} .
\end{aligned}
$$

Summing (21) and (22) and using the cancellation $I_{1}+J_{3}=$ 0 , due to Lemma 3 , we have

$$
\begin{gathered}
\frac{1}{2} \frac{d}{d t} \int|\Delta Q|^{2}+|\nabla u|^{2} d x+\int|\nabla \Delta Q|^{2}+|\Delta u|^{2} d x \\
=I_{2}+I_{3}+I_{4}+I_{5}+I_{6}+J_{1}+J_{2} .
\end{gathered}
$$

(i) Let (8) hold true. Using the integration by parts, $I_{2}$ can be bounded as

$$
\begin{aligned}
I_{2}= & \sum_{i} \int \partial_{i} Q \partial_{i}(\Omega \Delta Q)-\partial_{i} \Omega \partial_{i} Q \Delta Q-\Omega \partial_{i} Q \partial_{i} \Delta Q d x \\
\leq & C\|\nabla Q\|_{L^{q}}\|\Omega\|_{L^{2 q /(q-2)}}\|\nabla \Delta Q\|_{L^{2}} \\
& +C\|\nabla Q\|_{L^{q}}\|\nabla \Omega\|_{L^{2}}\|\Delta Q\|_{L^{2 q /(q-2)}}
\end{aligned}
$$

$$
\begin{aligned}
\leq & C\|\nabla Q\|_{L^{q}}\|\nabla u\|_{L^{2}}^{1-d / q}\|\Delta u\|_{L^{2}}^{d / q}\|\nabla \Delta Q\|_{L^{2}} \\
& +C\|\nabla Q\|_{L^{q}}\|\Delta u\|_{L^{2}}\|\Delta Q\|_{L^{2}}^{1-d / q}\|\nabla \Delta Q\|_{L^{2}}^{d / q} \\
\leq & C\|\nabla Q\|_{L^{q}}\left(\|\nabla u\|_{L^{2}}+\|\Delta Q\|_{L^{2}}\right)^{1-d / q} \\
& \times\left(\|\Delta u\|_{L^{2}}+\|\nabla \Delta Q\|_{L^{2}}\right)^{1+d / q} \\
\leq & \frac{1}{32}\|\Delta u\|_{L^{2}}^{2}+\frac{1}{32}\|\nabla \Delta Q\|_{L^{2}}^{2}+C\|\nabla Q\|_{L^{q}}^{p} \\
& \times\left(\|\nabla u\|_{L^{2}}^{2}+\|\Delta Q\|_{L^{2}}^{2}\right) .
\end{aligned}
$$

Here $p$ and $q$ satisfy the relation (8), and we have used the Gagliardo-Nirenberg inequality

$$
\|w\|_{L^{2 q /(q-2)}} \leq C\|w\|_{L^{2}}^{1-d / q}\|\nabla w\|_{L^{2}}^{d / q} .
$$

Similarly, we get

$$
\begin{aligned}
I_{3}, I_{4}, I_{5}, J_{2} \leq & \frac{1}{32}\|\Delta u\|_{L^{2}}^{2}+\frac{1}{32}\|\nabla \Delta Q\|_{L^{2}}^{2} \\
& +C\|\nabla Q\|_{L^{q}}^{p}\left(\|\nabla u\|_{L^{2}}^{2}+\|\Delta Q\|_{L^{2}}^{2}\right) .
\end{aligned}
$$

By using (16), $I_{6}$ is simply bounded as

$$
I_{6} \leq C\|\Delta Q\|_{L^{2}}^{2} .
$$

Here we treat the term

$$
\begin{aligned}
\int b \nabla Q \nabla Q \Delta Q d x & \leq C\|\nabla Q\|_{L^{4}}^{2}\|\Delta Q\|_{L^{2}} \\
& \leq C\|Q\|_{L^{\infty}}\|\Delta Q\|_{L^{2}}^{2} \leq C\|\Delta Q\|_{L^{2}}^{2}
\end{aligned}
$$

by the Gagliardo-Nirenberg inequality

$$
\|\nabla Q\|_{L^{4}}^{2} \leq C\|Q\|_{L^{\infty}}\|\Delta Q\|_{L^{2}} .
$$

Inserting the above estimates into (23), we derive

$$
\begin{aligned}
& \frac{1}{2} \frac{d}{d t} \int|\Delta Q|^{2}+|\nabla u|^{2} d x+\frac{1}{2} \int|\nabla \Delta Q|^{2}+|\Delta u|^{2} d x \\
& \quad \leq J_{1}+C\|\nabla Q\|_{L^{q}}^{p}\left(\|\nabla u\|_{L^{2}}^{2}+\|\Delta Q\|_{L^{2}}^{2}\right)+C\|\Delta Q\|_{L^{2}}^{2} .
\end{aligned}
$$

Now we estimate $J_{1}$ as follows.

(1) Let (5) hold true. We will use the following inequality [6]:

$$
\|u \cdot \nabla u\|_{L^{2}} \leq C\|u\|_{\dot{B}_{\infty, \infty}^{-s}}\|\nabla u\|_{\dot{H}^{s}},
$$

and the Gagliardo-Nirenberg inequality

$$
\|\nabla u\|_{\dot{H}^{s}} \leq C\|\nabla u\|_{L^{2}}^{1-s}\|\Delta u\|_{L^{2}}^{s} .
$$

Using (31) and (32), we bound $J_{1}$ as follows:

$$
\begin{aligned}
J_{1} & \leq\|u \cdot \nabla u\|_{L^{2}}\|\Delta u\|_{L^{2}} \leq C\|u\|_{\dot{B}_{\infty, \infty}^{-s}}\|\nabla u\|_{L^{2}}^{1-s}\|\Delta u\|_{L^{2}}^{s} \\
& \leq \frac{1}{16}\|\Delta u\|_{L^{2}}^{2}+C\|u\|_{\dot{B}_{\infty, \infty}^{-s}}^{2 /(1-s)}\|\nabla u\|_{L^{2}}^{2} .
\end{aligned}
$$


Substituting the above estimates into (30), we reach

$$
\begin{gathered}
\|u\|_{L^{\infty}\left(0, T ; H^{1}\right)}+\|u\|_{L^{2}\left(0, T ; H^{2}\right)} \leq C, \\
\|Q\|_{L^{\infty}\left(0, T ; H^{2}\right)}+\|Q\|_{L^{2}\left(0, T ; H^{3}\right)} \leq C .
\end{gathered}
$$

This completes the proof.

(2) Let (6) hold true. Using the following elegant inequality $[17,18]$ :

$$
\|\nabla u\|_{L^{4}}^{2} \leq C\|u\|_{\dot{B}_{\infty, \infty}^{0}}\|\Delta u\|_{L^{2}}
$$

we bound $J_{1}$ as follows:

$$
\begin{aligned}
J_{1} & =\sum_{i, j} \int u_{i} \partial_{i} u \partial_{j}^{2} u d x=-\sum_{i, j} \int \partial_{j} u_{i} \partial_{i} u \partial_{j} u d x \\
& \leq C\|\nabla u\|_{L^{4}}^{2}\|\nabla u\|_{L^{2}} \leq C\|u\|_{\dot{B}_{\infty, \infty}^{0}}\|\nabla u\|_{L^{2}}\|\Delta u\|_{L^{2}} \\
& \leq \frac{1}{16}\|\Delta u\|_{L^{2}}^{2}+C\|u\|_{\dot{B}_{\infty, \infty}^{0}}^{2}\|\nabla u\|_{L^{2}}^{2} .
\end{aligned}
$$

Substituting the above estimates into (30), we have (34).

This completes the proof.

(3) Let (7) hold true. Let $\left\{\phi_{j}\right\}_{j \in \mathbb{Z}}$ be the Littlewood-Paley dyadic decomposition of unity that satisfies $\widehat{\phi} \in C_{0}^{\infty}\left(B_{2} \backslash\right.$ $\left.B_{1 / 2}\right), \widehat{\phi}_{j}(\xi)=\widehat{\phi}\left(2^{-j} \xi\right)$, and $\sum_{j \in \mathbb{Z}} \widehat{\phi}_{j}(\xi)=1$ for any $\xi \neq 0$, where ${ }^{-}$is the Fourier transform and $B_{r}$ is the ball with radius $r$ centered at the origin.

We decompose $\partial_{j} u$ as follows:

$$
\begin{aligned}
\partial_{j} u= & \sum_{k=-\infty}^{\infty} \phi_{k} * \partial_{j} u=\sum_{k<-N} \phi_{k} * \partial_{j} u+\sum_{k=-N}^{N} \phi_{k} * \partial_{j} u \\
& +\sum_{k>N} \phi_{k} * \partial_{j} u,
\end{aligned}
$$

where $N$ is a positive integer to be chosen later. Plugging this decomposition into $J_{1}$, we derive

$$
\begin{gathered}
J_{1}=-\sum_{i, j} \int \partial_{j} u_{i} \partial_{i} u\left(\sum_{k<-N} \phi_{k} * \partial_{j} u+\sum_{k=-N}^{N} \phi_{k} * \partial_{j} u\right. \\
\left.+\sum_{k>N} \phi_{k} * \partial_{j} u\right) d x \\
=: J_{11}+J_{12}+J_{13} .
\end{gathered}
$$

Recalling Bernstein's inequality,

$$
\left\|\phi_{k} * f\right\|_{L^{q}} \leq C 2^{d k(1 / p-1 / q)}\left\|\phi_{k} * f\right\|_{L^{p}}, \quad 1 \leq p \leq q \leq \infty,
$$

with $C$ being a positive constant independent of $f$ and $k$, we apply Hölder's inequality to deduce that

$$
\begin{aligned}
& J_{11} \leq \sum_{i, j}\left\|\partial_{j} u_{i}\right\|_{L^{2}}\left\|\partial_{i} u\right\|_{L^{2}} \sum_{k<-N}\left\|\phi_{k} * \partial_{j} u\right\|_{L^{\infty}} \\
& \leq C\|\nabla u\|_{L^{2}}^{2} \sum_{k<-N} 2^{(d / 2) k}\left\|\phi_{k} * \nabla u\right\|_{L^{2}} \\
& \leq C 2^{-(d / 2) N}\|\nabla u\|_{L^{2}}^{3} \text {, } \\
& J_{12} \leq \sum_{i, j}\left\|\partial_{j} u_{i}\right\|_{L^{2}}\left\|\partial_{i} u\right\|_{L^{2}} \sum_{k=-N}^{N}\left\|\phi_{k} * \partial_{j} u\right\|_{L^{\infty}} \\
& \leq C\|\nabla u\|_{L^{2}}^{2} \cdot N\|\nabla u\|_{\dot{B}_{\infty, \infty}^{0}} \\
& J_{13} \leq \sum_{i, j}\left\|\partial_{j} u_{i}\right\|_{L^{3}}\left\|\partial_{i} u\right\|_{L^{3}} \sum_{k>N}\left\|\phi_{k} * \partial_{j} u\right\|_{L^{3}} \\
& \leq C\|\nabla u\|_{L^{3}}^{2} \sum_{k>N} 2^{(d / 6) k}\left\|\phi_{k} * \nabla u\right\|_{L^{2}} \\
& \leq C\|\nabla u\|_{L^{3}}^{2}\left(\sum_{k>N} 2^{2(d / 6-1) k}\right)^{1 / 2} \\
& \times\left(\sum_{k>N} 2^{2 k}\left\|\phi_{k} * \nabla u\right\|_{L^{2}}^{2}\right)^{1 / 2} \\
& \leq C\|\nabla u\|_{L^{3}}^{2} 2^{-(1-(d / 6) N)}\|\Delta u\|_{L^{2}} \\
& \leq C 2^{-(1-d / 6) N}\|\nabla u\|_{L^{2}}^{2(1-d / 6)}\|\Delta u\|_{L^{2}}^{1+d / 3} .
\end{aligned}
$$

Now we choose $N$ so that $C 2^{-(1-d / 6) N}\|\nabla u\|_{L^{2}}^{2(1-d / 6)} \leq 1 / 16$ and $2^{-(d / 2) N}\|\nabla u\|_{L^{2}} \leq 1 / 16$ to conclude that

$$
\begin{aligned}
J_{1} \leq & C\|\nabla u\|_{L^{2}}^{2}+C\|\nabla u\|_{L^{2}}^{2}\|\nabla u\|_{\dot{B}_{\infty, \infty}^{0}} \log \left(e+\|\nabla u\|_{L^{2}}+\|\Delta Q\|_{L^{2}}\right) \\
& +\frac{1}{16}\|\Delta u\|_{L^{2}}^{2}+C .
\end{aligned}
$$

Substituting the above estimates into (30), we arrive at (34).

This completes the proof of part (i).

(ii) Let (9) hold true. We still have (23).

$I_{2}$ is simply bounded as

$$
\begin{aligned}
I_{2} & \leq C\|\Omega\|_{L^{q}}\|\Delta Q\|_{L^{2 q /(q-1)}}^{2} \\
& \leq C\|\nabla u\|_{L^{q}}\|\Delta Q\|_{L^{2}}^{2(1-3 / 2 q)}\left\|\nabla^{3} Q\right\|_{L^{2}}^{3 / q} \\
& \leq \frac{1}{32}\left\|\nabla^{3} Q\right\|_{L^{2}}^{2}+C\|\nabla u\|_{L^{q}}^{p}\|\Delta Q\|_{L^{2}}^{2} .
\end{aligned}
$$

Here we have used the Gagliardo-Nirenberg inequality

$$
\|w\|_{L^{2 q /(q-1)}} \leq C\|w\|_{L^{2}}^{1-3 / 2 q}\|\nabla w\|_{L^{2}}^{3 / 2 q} .
$$


$I_{3}$ is simply bounded as

$$
\begin{aligned}
I_{3} & =-2 \sum_{i} \int\left(\Omega \partial_{i}\left(\partial_{i} Q \Delta Q\right)-\partial_{i}^{2} Q \Omega \Delta Q-\partial_{i} Q \Omega \partial_{i} \Delta Q\right) d x \\
& \leq C\|\Omega\|_{L^{q}}\left(\|\Delta Q\|_{L^{2 q /(q-1)}}^{2}+\|\nabla Q\|_{L^{2 q /(q-2)}}\left\|\nabla^{3} Q\right\|_{L^{2}}\right) \\
& \leq C\|\nabla u\|_{L^{q}}\|\Delta Q\|_{L^{2}}^{2(1-3 / 2 q)}\left\|\nabla^{3} Q\right\|_{L^{2}}^{3 / q} \\
& \leq \frac{1}{32}\left\|\nabla^{3} Q\right\|_{L^{2}}^{2}+C\|\nabla u\|_{L^{q}}^{p}\|\Delta Q\|_{L^{2}}^{2} .
\end{aligned}
$$

Here we have used (43) and the Gagliardo-Nirenberg inequality

$$
\|w\|_{L^{2 q /(q-2)}} \leq C\|\nabla w\|_{L^{2}}^{2-3 / q}\|\Delta w\|_{L^{2}}^{3 / q-1}
$$

Similarly, $I_{4}, I_{5}, J_{2}$, and $J_{1}$ can be bounded as follows:

$$
\begin{aligned}
I_{4}+I_{5}+J_{2} & \leq C\|\nabla u\|_{L^{q}}\left(\|\Delta Q\|_{L^{2 q /(q-1)}}^{2}+\|\nabla Q\|_{L^{2 q /(q-2)}}\left\|\nabla^{3} Q\right\|_{L^{2}}\right) \\
& \leq C\|\nabla u\|_{L^{q}}\|\Delta Q\|_{L^{2}}^{2-3 / q}\left\|\nabla^{3} Q\right\|_{L^{2}}^{3 / q} \\
& \leq \frac{1}{32}\left\|\nabla^{3} Q\right\|_{L^{2}}^{2}+C\|\nabla u\|_{L^{q}}^{p}\|\Delta Q\|_{L^{2}}^{2}, \\
J_{1} & =-\sum_{i, j} \int \partial_{i} u_{j} \partial_{i} u \partial_{j} u d x \\
& \leq C\|\nabla u\|_{L^{q}}\|\nabla u\|_{L^{2 q /(q-1)}}^{2} \\
& \leq C\|\nabla u\|_{L^{q}}\|\nabla u\|_{L^{2}}^{2(1-3 / 2 q)}\|\Delta u\|_{L^{2}}^{3 / q} \\
& \leq \frac{1}{32}\|\Delta u\|_{L^{2}}^{2}+C\|\nabla u\|_{L^{q}}^{p}\|\nabla u\|_{L^{2}}^{2} .
\end{aligned}
$$

$I_{6}$ is bounded as above.

Inserting the above estimates into (23), using the Gronwall inequality, we arrive at (34).

This completes the proof.

\section{Acknowledgments}

This paper is supported by NSFC (no. 11171154). The authors are indebted to the referee for nice suggestions which improved the paper.

\section{References}

[1] A. N. Beris and B. J. Edwards, Thermodynamics of Flowing Systems with Internal Microstructure, vol. 36 of Oxford Engineering Science Series, Oxford University Press, Oxford, UK, 1994.

[2] C. Denniston, E. Orlandini, and J. M. Yeomans, "Lattice Boltzmann simulations of liquid crystal hydrodynamics," Physical Review E, vol. 63, no. 5, pp. 567021-5670210, 2001.

[3] P. G. De Gennes, The Physics of Liquid Crystals, Clarendon Press, Oxford, UK, 1974.
[4] G. Tóth, C. Denniston, and J. M. Yeomans, "Hydrodynamics of domain growth in nematic liquid crystals," Physical Review E, vol. 67, no. 5, Article ID 051705, 11 pages, 2003.

[5] H. Kozono, T. Ogawa, and Y. Taniuchi, "The critical Sobolev inequalities in Besov spaces and regularity criterion to some semi-linear evolution equations," Mathematische Zeitschrift, vol. 242, no. 2, pp. 251-278, 2002.

[6] H. Kozono and Y. Shimada, "Bilinear estimates in homogeneous Triebel-Lizorkin spaces and the Navier-Stokes equations," Mathematische Nachrichten, vol. 276, pp. 63-74, 2004.

[7] H. Triebel, Theory of Function Spaces, Birkhäuser, Basel, Switzerland, 1983.

[8] M. Paicu and A. Zarnescu, "Energy dissipation and regularity for a coupled Navier-Stokes and Q-tensor system," Archive for Rational Mechanics and Analysis, vol. 203, pp. 45-67, 2012.

[9] F. M. Leslie, "Some constitutive equations for liquid crystals," Archive for Rational Mechanics and Analysis, vol. 28, no. 4, pp. 265-283, 1968.

[10] F. H. Lin and C. Liu, "Nonparabolic dissipative systems modeling the flow of liquid crystals," Communications on Pure and Applied Mathematics, vol. 48, no. 5, pp. 501-537, 1995.

[11] F. H. Lin and C. Liu, "Partial regularity of the dynamic system modeling the flow of liquid crystals," Discrete and Continuous Dynamical Systems, vol. 2, no. 1, pp. 1-22, 1996.

[12] F. H. Lin and C. Liu, "Existence of solutions for the EricksenLeslie system," Archive for Rational Mechanics and Analysis, vol. 154, no. 2, pp. 135-156, 2000.

[13] Z. Lei, N. Masmoudi, and Y. Zhou, "Remarks on the blowup criteria for Oldroyd models," Journal of Differential Equations, vol. 248, no. 2, pp. 328-341, 2010.

[14] J. Fan and B. Guo, "Regularity criterion to some liquid crystal models and the Landau-Lifshitz equations in $\mathbb{R}^{3}$," Science in China A, vol. 51, no. 10, pp. 1787-1797, 2008.

[15] J. Fan and T. Ozawa, "Regularity criteria for a simplified Ericksen-Leslie system modeling the flow of liquid crystals," Discrete and Continuous Dynamical Systems, vol. 25, no. 3, pp. 859-867, 2009.

[16] M. H. Giga, Y. Giga, and J. Saal, Nonlinear Partial Differential Equations, Birkhäauser, 2010.

[17] S. Machihara and T. Ozawa, "Interpolation inequalities in Besov spaces," Proceedings of the American Mathematical Society, vol. 131, no. 5, pp. 1553-1556, 2003.

[18] Y. Meyer, "Oscillating patterns in some nonlinear evolution equations," Lecture Notes in Mathematics, vol. 1871, pp. 101-187, 2005. 


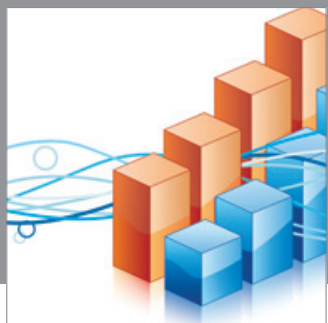

Advances in

Operations Research

mansans

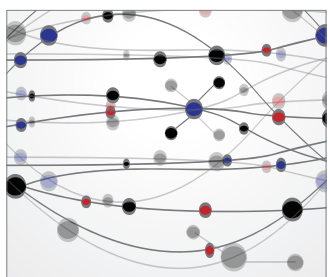

The Scientific World Journal
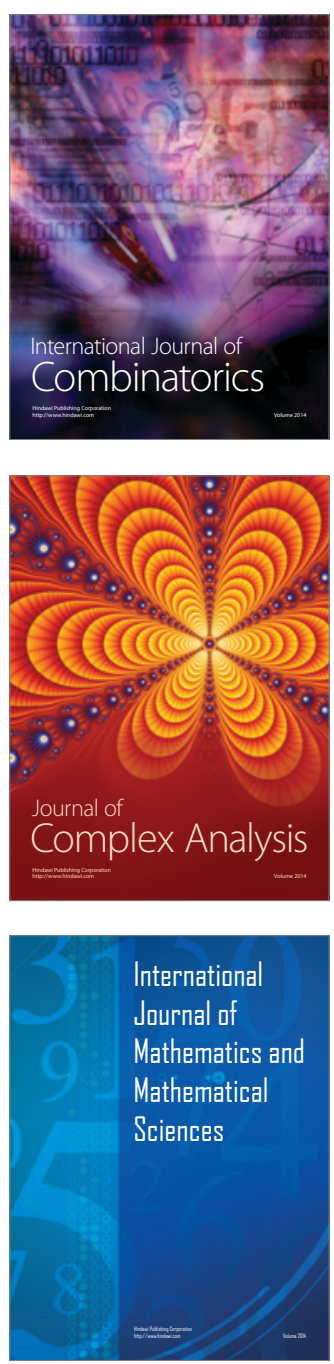
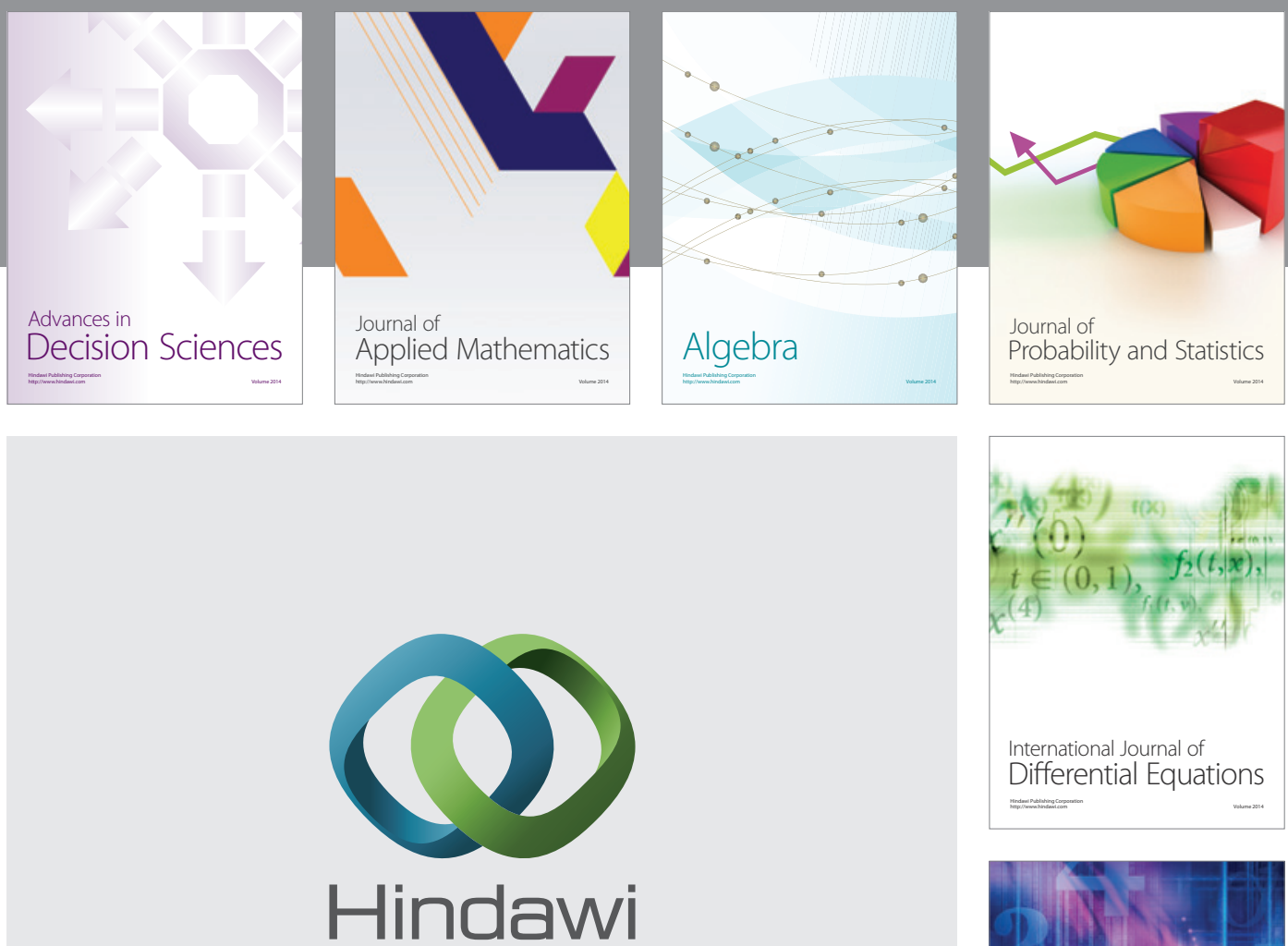

Submit your manuscripts at http://www.hindawi.com
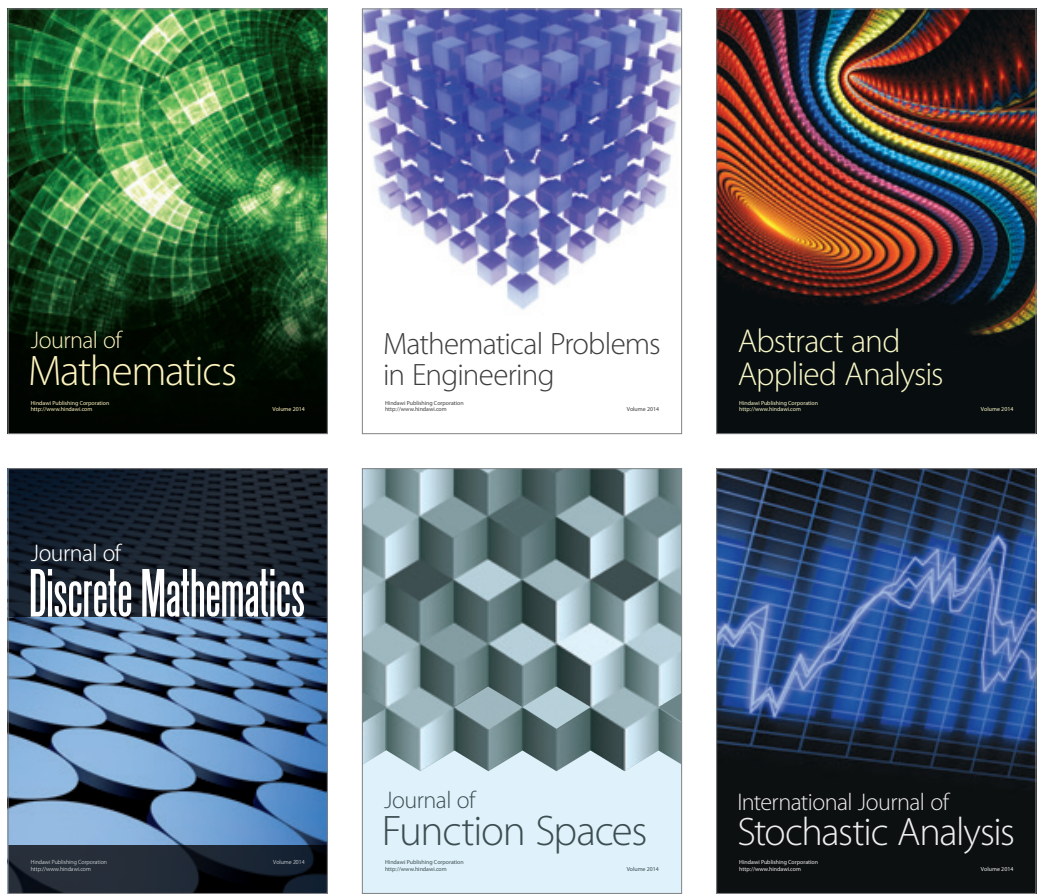

Journal of

Function Spaces

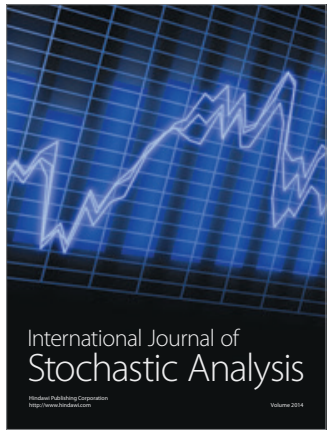

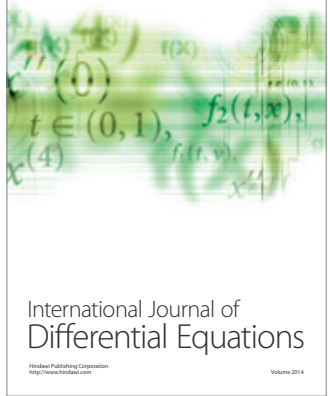
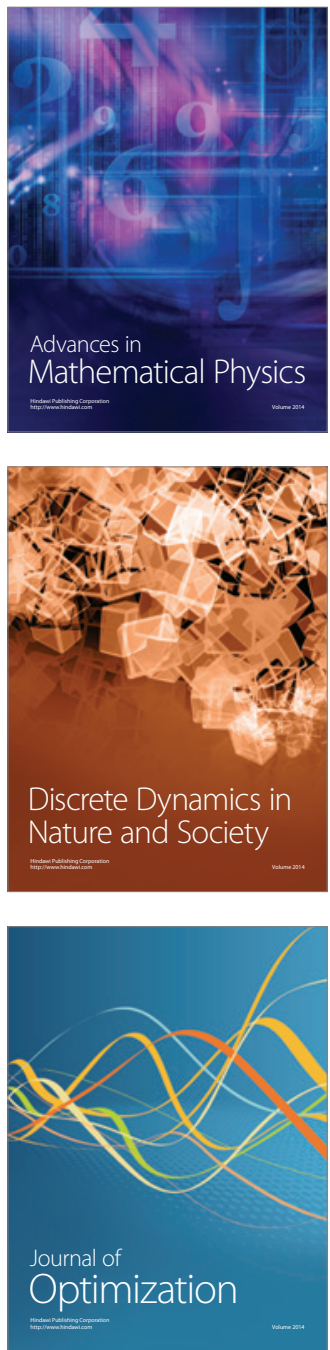